\title{
Use of a Modified Richardson Number for Parameterizing the Effect of Shallow Convection
}

\author{
By JF. Geleyn \\ Direction de la Météorologie Nationale, EERM/CRMD Paris, France \\ (Manuscript received 4 November 1986, in revised form 3 February 1987)
}

\begin{abstract}
In the framework of a $K$-exchange parameterization for PBL turbulent fluxes we propose a simple way to include the effects of shallow convective clouds. The method is dimensionally consistent with cloud buoyancy production, is cheap and avoids the introduction of additional arbitrary parameters by simply extending the dependency of the exchange coefficient on the Richardson number from the dry to the moist case.

In the case of the french large scale operational model it is very efficient in suppressing the defects associated with the PBL collapse that is usually observed when one uses only "dry" $K$-coefficients.
\end{abstract}

\section{Introduction}

This paper describes the method that is used in the French Weather Service's operational models to overcome the difficulties associated with the use of "dry-computed" $K$ coefficients in a multi-level/exchange-coefficients parameterization of $\mathrm{PBL}$ processes. These difficulties are linked to the non-simulation of enhanced vertical exchange at the top of the PBL in presence of shallow convective clouds and the method used to remove them is commonly refered to as "parameterization of shallow convection". We shall comply to this convention from now on. It should be stressed that, since we want to stick to the $K$-coefficient approach for several independant reasons, we shall not discuss here the relative merits of the exchange coefficient approach and of the bulk PBL approach with regards to the introduction of shallow convection parameterization. In Part II we shall explain the need for a specific parameterization of shallow convection in $K$-exchange-type formulations; in Part III we shall review several concrete approaches to solve the problem, indicate their relative advantages and disad- vantages, and therefore draw a list of desirable properties for such a parameterization; our proposal will be the subject of Part IV. Finally we shall show the experimental behaviour of that solution in Part V.

\section{The problem}

During the last ten years several parameterizations of the vertical exchange in the planetary boundary layer have been developed that make explicit use of the local Richardson number $R_{i}$ to compute vertical exchange coefficients $K$ following:

$$
K=l^{2}(z)\left|\frac{\partial \vec{u}}{\partial z}\right| f\left(R_{i}\right)
$$

with

$$
R_{i}=\frac{g}{C_{p} T} \frac{\partial s / \partial z}{|\partial \vec{u} / \partial z|^{2}}
$$

$s$ being the dry static energy $C_{p} T+g z$.

The choice of the formulae for $l(z)$ and $f\left(R_{i}\right)$ is out of the scope of this paper. Let us simply say that all the experiments shown hereafter were done with the 1983 version of the ECMWF scheme as described by Louis, Tiedtke and Geleyn (1982), version that is implemented in the French operational models 
"Emeraude" (Coiffier et al. 1987) and "Peridot" (Imbard et al. 1987) (respectively hemispheric/ large-scale and limited area/fine mesh).

This type of parameterization scheme leads to good simulations of the PBL fluxes as long as no clouds are present. But, as noticed, among others, by Tiedtke (1982) a problem arises as soon as a cloud develops at the top of the boundary layer underneath the inversion. To show this problem let us consider four locations across the depth of the lower atmosphere: the dry well mixed layer (A); the cloud layer (B); the thin inversion slab (C); the "free atmosphere" (D). We shall now describe in Table 1 what happens when only the "dry" version of the PBL scheme (as represented by Equations (1) and (2)) is used:

Table 1 Characteristics of the "dry" PBL scheme as represented by Eqs. (1) and (2).

\begin{tabular}{|c|c|c|c|c|}
\hline & $\begin{array}{l}\text { A the dry well } \\
\text { mixed layer }\end{array}$ & $\mathrm{B}$ the cloud layer & $\begin{array}{l}\mathrm{C} \text { the thin } \\
\text { inversion slab }\end{array}$ & $\begin{array}{l}\text { D the "free } \\
\text { atmosphere" }\end{array}$ \\
\hline Richardson Number & Zero & $\begin{array}{l}\text { Moderate positive } \\
\text { value }\end{array}$ & $\begin{array}{l}\text { Very high positive } \\
\text { value }\end{array}$ & $\begin{array}{l}\text { Moderate positive } \\
\text { value }\end{array}$ \\
\hline $\begin{array}{l}\text { Exchange } \\
\text { coefficient }\end{array}$ & $\begin{array}{l}\text { Important and in- } \\
\text { creasing linearly } \\
\text { with height }\end{array}$ & Small but not zero & Zero & Small but not zero \\
\hline $\begin{array}{l}\text { Vertical moisture } \\
\text { flux }\end{array}$ & $\begin{array}{l}\text { Constant with } \\
\text { height and with a } \\
\text { value depending on } \\
\text { surface evaporation }\end{array}$ & $\begin{array}{l}\text { Decreasing with } \\
\text { height }\end{array}$ & Zero & Small but not zero \\
\hline Moisture tendency & Zero & $\begin{array}{l}\text { Increasingly posi- } \\
\text { tive with height }\end{array}$ & $\begin{array}{l}\text { Strongly positive } \\
\text { just underneath and } \\
\text { partly compensated } \\
\text { by rainfall drying }\end{array}$ & Small and negative \\
\hline $\begin{array}{l}\text { Temperature } \\
\text { tendency }\end{array}$ & Zero & Slightly negative & $\begin{array}{l}\text { Positive just under- } \\
\text { neath owing to the } \\
\text { latent heat release } \\
\text { associated with } \\
\text { moisture conver- } \\
\text { gence }\end{array}$ & Small and positive \\
\hline
\end{tabular}
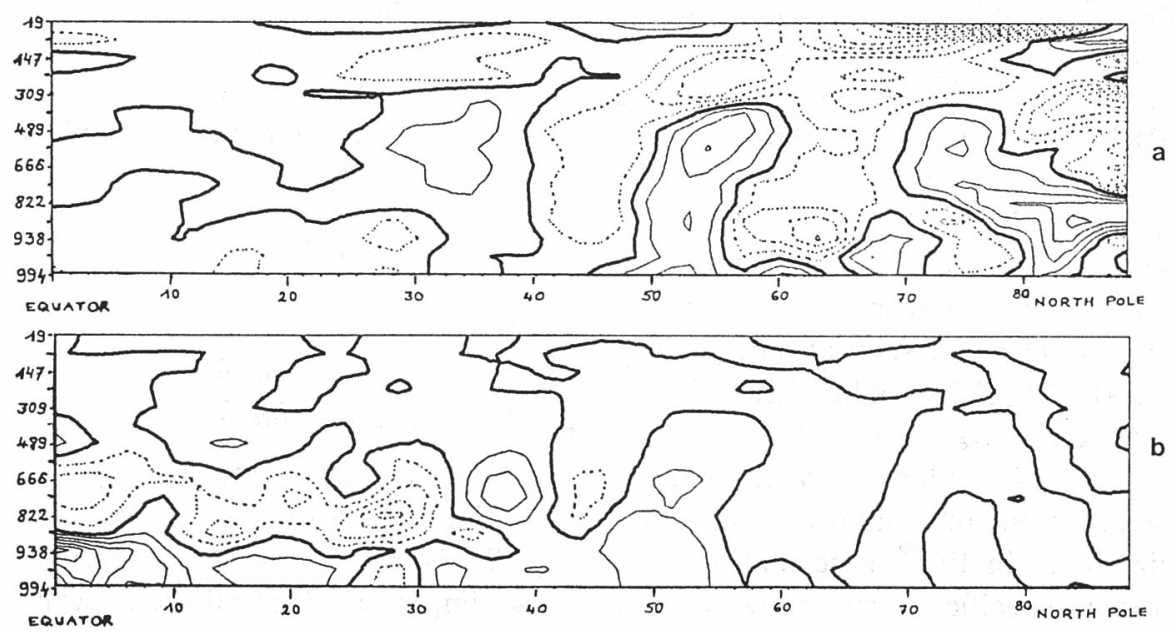

Fig. 1 Zonal means of temperature tendencies (a) and of specific humidity tendencies (b) between $24 \mathrm{~h}$ and $48 \mathrm{~h}$ during the model integration from 10-01-1986 at $00 \mathrm{Z}$ ( -0 line, positive values, $\cdots \cdots$ negative values, contour intervals are $: .5^{\circ} \mathrm{K} /$ day and $.2 \mathrm{~g} . \mathrm{kg}^{-1} /$ day (b) ; case without shallow convection parameterization. 
One can deduce that the following consequences will be observed in the model:

-the boundary layer gets moister and moister

- the free atmosphere gets dryer and dryer

- the top of the boundary layer collapses as heating underneath brings the inversion down.

Figure 1 shows quite well such a behaviour in our hemispheric model through the diagnostic of total zonal mean tendencies for temperature (a) and specific humidity (b): in the tropics and subtropics the contrast between PBL moistening and free atmosphere drying is obvious. Notice that feed-back mechanisms (radiative and dynamical) have greatly compensated for the temperature effect.

Of course all this does not happen in nature. Instead the turbulence generated by the cloud sitting underneath the inversion transports moisture towards the free atmosphere (and obviously heat and momentum as well). So the problem can now be formulated as follows: how can we simulate the exchange taking place across the inversion when the moisture structure of the model suggests the presence of a cloud there?

\section{Possible solutions}

Remembering that all the following only refers to $K$-theory PBL parameterizations we look back at Equations 1 and 2. There are obviously two ways of increasing the exchange, either by directly enhancing $K$ or by decreasing $R_{i}$ and letting the $f$ function work on the $K$ increase. In both cases the condensational heating and drying in the lower cloud layer and the evaporative cooling and moistening in the upper part of the cloud cannot be explicitely accounted for. However this may be a minor drawback since they compensate each other and since several other causes of vertical redistribution are working at the same time and probably with a stronger intensity in the full ensemble of parameterizations.

The first solution ( $K$ enhancement) was successfully applied by Tiedtke (1984) to the
ECMWF model: one assumes a PBL cloud when the lifting condensation level is underneath a diagnosed inversion. The "dry" computed $K$ is then increased by three arbitrarily chosen amounts at the bottom, inside and at the top of the cloud, respectively. This method brings good results but suffers, in our opinion, of several drawbacks. It is not easy to implement numerically (apparently the nonl inear behaviour of the pseudo-implicit solution for the vertical diffusion equations makes it necessary to first solve for the dry case before solving a second time for the increments of $K$ ), the tuning of the arbitrary parameters might be very much modeldependent and, most essentially, the continuous dependency of the process on the atmospheric parameters that is implicitly contained in any parameterization using the solution of Equation (1) gets lost in this treatment.

However these drawbacks may be price to pay to get the freedom to choose a non-local closure for the parameterization of shallow convection. Although the effect we wish to parameterize is a mixing of subcloud air and of air above the level of non-buoyancy, its intensity seems indeed to depend mostly on subcloud layer turbulence (Le Mone and Pennel, 1976) and little on the thermal conditions above cloud base. Thus the $K$-approach seems to offer a more direct way of taking into account the physics of the process but the simplicity of the Richardson number approach might more than compensate for this advantage if feed-back processes are allowed that may lead to the same atmospheric effects for different numerical reasons. We shall come back on this point in Part V.

If one therefore turns to the modification of $R_{i}$, one can find several proposals, the first one, associated with the development of the ECMWF formulation, probably being the one of Louis (1977). They are all more or less based upon the diagnostic of what physically happens inside the cloud: the latent heat release modifies the buoyancy production that is the upper case term in the Richardson number. So, if the cloud covers the whole grid point area, replacing $s$ by $h_{s}=C_{p} T+g z$ $+L q_{s}$ in Equation (2) (with $q_{s}$ saturation 
specific humidity and $h_{s}$ moist saturated static energy) will lead to a set of equations for the moist turbulent vertical exchange that is the exact pendant of the previous one for the dry case. Even if the cloud is only covering a partial area in the ratio $C$, the replacement of $s$ by $C_{p} T+g z+C L q_{s}$ will also be consistent, but only as long as $C$ remains constant with height.

There appears the reason why such a "physical" method is encountering many difficulties when applied to a discretised situation. At the bottom of the cloud the partial cover will have to jump from zero to $C$ and the reverse will happen at the top of the cloud. Thus, simply comparing $C q_{s}$ inside the cloud with zero outside is of course meaningless and dangerous. Furthermore, one needs some way to compute $C$, usually from the ratio $q / q_{s}$ (relative humidity). We tried a number of ad-hoc formulations that were adding to $\partial s / \partial z$ a quantity dimensionally equivalent to $\partial\left(C L q_{s}\right) / \partial z$ but for which the problem of top and bottom discontinuities was smoothed out. For all of them but one, either nothing was happening or, more often, an uncontrolled PBL growth was observed, in opposition to the collapse explained in Part II. Such a behaviour is documented in Figure 2 for the same case as Figure 1, $s$ being simply replaced by $h=s+L q$ (moist static energy) in Equation 2. One notices on the temperature diagram that the $\mathrm{PBL}$ is still warming at
$48 \mathrm{~h}$ range and reaches up to $400 \mathrm{hPa}$ ! What happens is the following: the increase of $K$ brings humidity across the inversion but the strong gradient of $q$ is simply displaced one model layer higher up. So, as soon as enough moisture has been supplied by surface evaporation, the process starts again at a new level, quite regardless of the atmospheric thermal structure. This will only stop at the detrainment height of deep convection! The fact that the maximum gradient of $q$ and the high values of $K$ are following each other upwards indicates that this approach lacks a selfregulating mechanism to prevent such an absurd numerical behaviour.

In summary, to solve the problem without by-passing Equation 1, without obtaining an uncontrolled PBL growth and with as physical a solution as possible, we want:

-a modification of $R_{i}$ through some addition to $\partial s / \partial z$.

-no arbitrary parameter and the same "dimensional" behaviour as $C \cdot \partial\left(L q_{s}\right) / \partial z$ (or $\left.\partial\left(C L q_{s}\right) / \partial z\right)$ for this addition.

-self-regulating properties for the scheme.

\section{Proposal}

All the above mentioned properties are obtained by the following formulation:

$$
R_{i}^{*}=\frac{g}{C_{p} T} \frac{\partial s / \partial z+L \cdot \min \left(0, \partial\left(q-q_{s}\right) / \partial z\right)}{|\partial \vec{u} / \partial z|^{2}}
$$
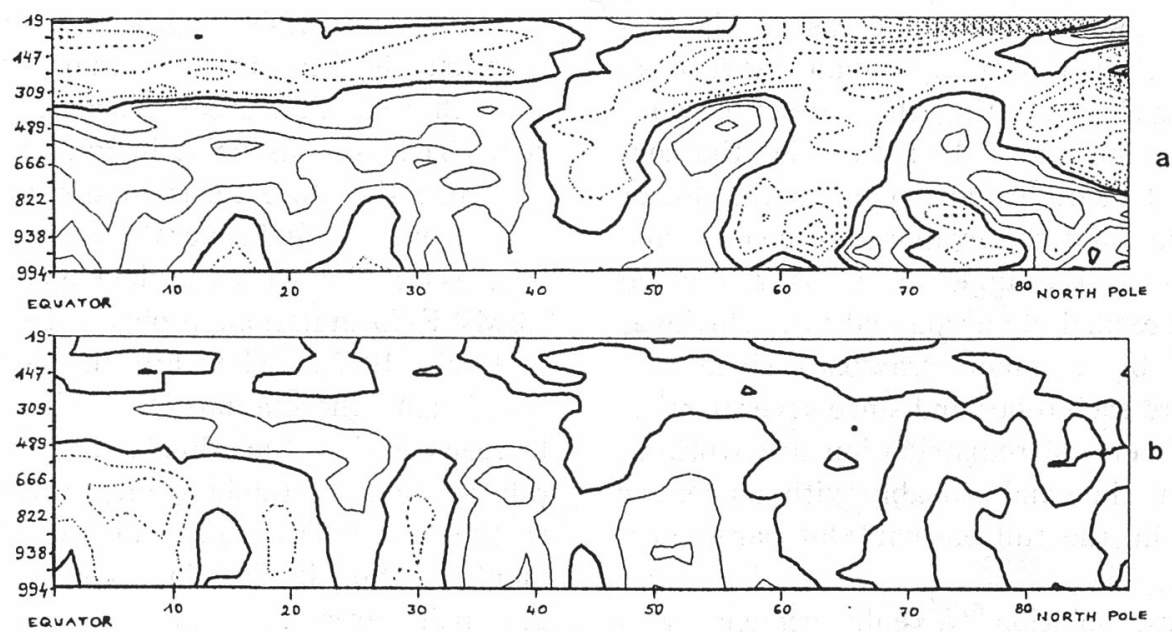

Fig. 2 As Figure 1 except for: case with an unregulated shallow convection scheme. 
At first sight the self-regulating aspect is the only non obvious feature of this choice. However it is easy to verify that any increase of the vertical exchange will tend towards near zero gradients of $q$ and $s$, therefore towards a negative vertical gradient of $q_{s}$ (via $\left.\partial T / \partial z=-g / C_{p}\right)$ and thus the choice of the "zero option" by the function "min" in Equation 3 will be obtained before the exchange would be total. Therefore a balance between the dynamically maintained strength of the inversion and the exchange across it will be observed in the model. One might ask whether an undiscriminating use of the same $K$ determination from the surface up into the free atmosphere is physically sound. The answer to that question can only be: no more but no less than in the dry case; i.e. it is part of the acceptance of a $K$-coefficient PBL parameterization to seek as general and as continuous formulations as possible to try and successfully incompass a huge variety of observed vertical structures and their evolutions into a single, albeit partly inadequate, framework.

The success of this formulation in removing the symptoms of the PBL collapse without leading to an uncontrolled growth is indicated in Figure 3 (to be compared with Figures 1 and 2); the tropical/subtropical belt is now nearly in equilibrium for $T$ and $q$ and the horizontally orientated structure of the moisture diagram has been replaced by ver- tically orientated ones.

Before going to the presentation of further results for this scheme let us address the question of the ad-hoc character of our formulation. One may ask whether the vertical gradient of $q-q_{s}$ has got some physical bearing on our original problem. The answer is twofold: no, there seems to be no way to derive a physically consistent variant of Equation 2 that leads to such a gradient; but yes, this is a good diagnostic parameter for an inversion and, intuitively, if we assume that the rest of the model's physics will tend towards moist adiabatic adjustment, only superadiabatic cases will require enhanced vertical diffusion. The apparent implication (by Equation 3) that the convective processes depend entirely on local gradients seems in contradiction with observational evidence showing the relation between the intensity of shallow convection and subcloud turbulence. However one should not forget that (a) the PBL set of diffusion equations will be numerically solved by an implicit algorithm (thus with non local properties) and that (b) all the feed-back processes allowed by the general and continuous caracter of our set of equations might (and at least partly do) work within several model time steps towards a realistic behaviour of the parameterized PBL. The results presented below strongly support this statement.
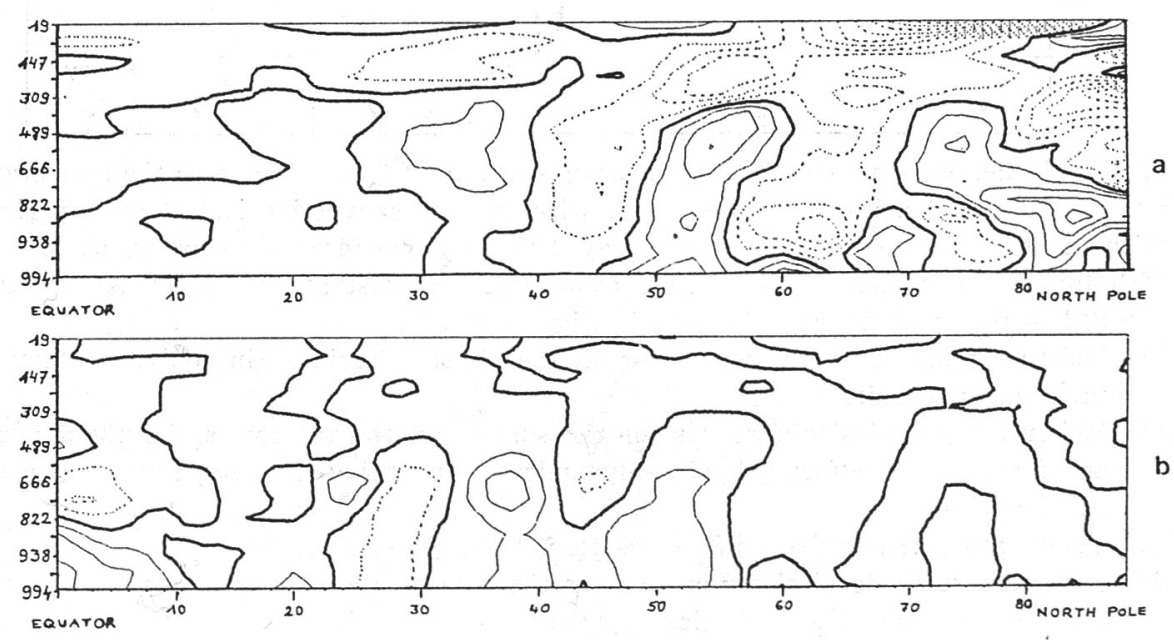

Fig. 3 As Fig. 1 except for: case with the proposed selfregulated shallow convection scheme. 

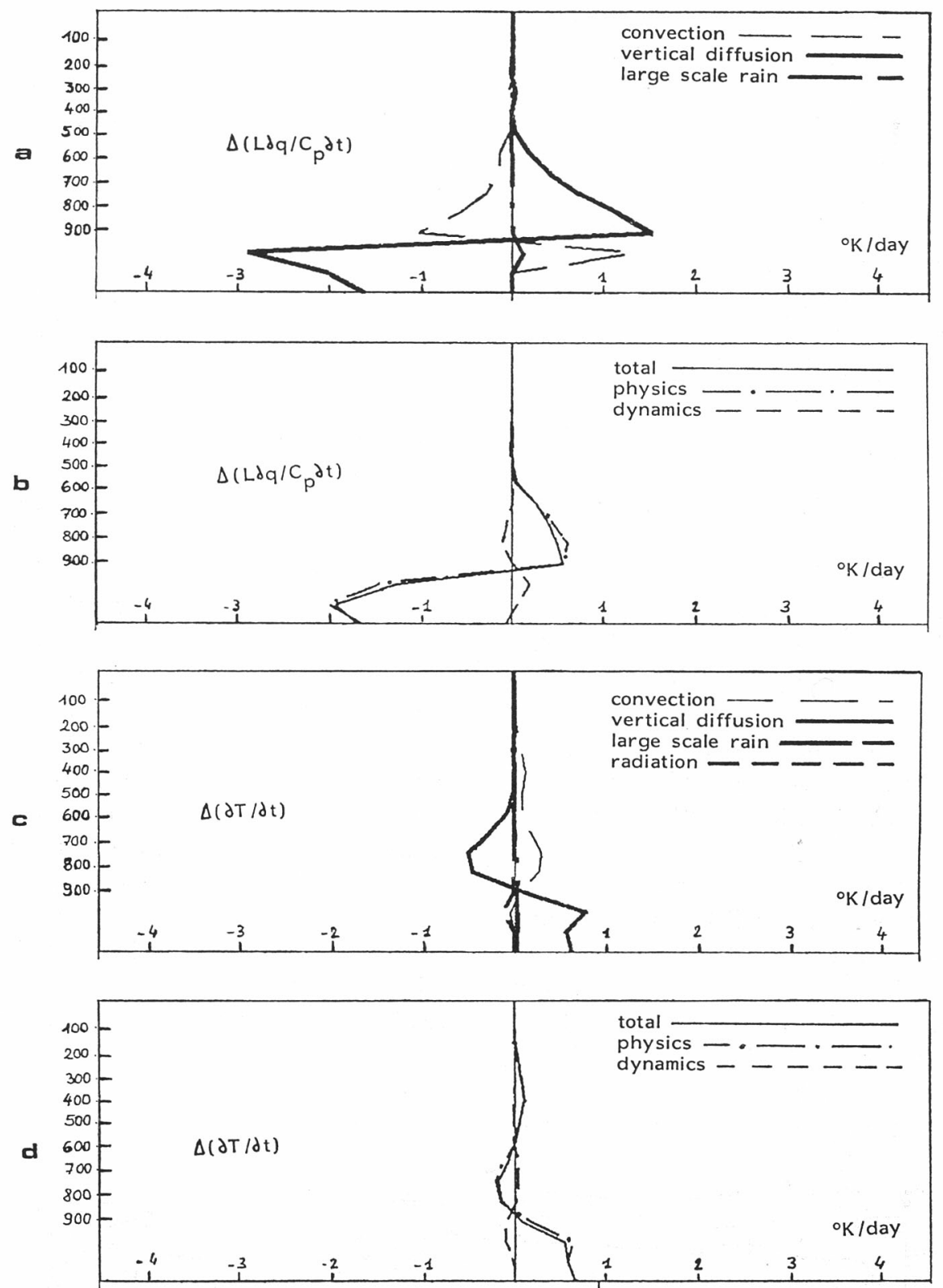

Fig. 4 Hemispherically averaged vertical profiles of the differences between the temperature and moisture tendencies of an experiment with shallow convection and of an experiment without it. Time averaging period: day 3 to 4 of two forecasts starting on May the 16th 1986. The choice of the vertical coordinate (equidistant model levels) stretches the PBL - that was already the case in Figs. 1 to 3 -.

a) Moisture tendencies' differences for all the physics forcings (in equivalent temperature tendency units);

b) Moisture tendencies' differences for the sum of all the (a) terms, for the dynamics and for the total effect (physics+dynamics) (in equivalent temperature tendency units);

c) Temperature tendencies' differences for all the physics forcing;

d) Temperature tendencies' differences for the sum of all (c) terms, for the dynamics and for the total effect (physics + dynamics).

Unit ${ }^{\circ} \mathrm{K} /$ day. 


\section{Results}

We shall look in more details at the differences between two integrations starting from May the 16th 1986, the first one with shallow convection parameterized according to equation 3 , the second one without such a parameterization, both being performed, as previously, with the 15 level/T79 version of our hemispheric model.

Figure 4 shows hemispheric averages of differences between specific humidity tendencies (a-b) and temperature tendencies (c-d) for the various physical terms $(a-c)$ and for the total physical and dynamical terms and their sum (b-d). Thus this figure shows the direct averaged impact of shallow convection taken in this case between day 3 and day 4 in the forecast. Two important facts are worth mentioning:

-about half of the moisture supplied to the free atmosphere by the shallow convection process is used to feed deep convection, the other half being of course recycled in the trade wind circulation.

-whereas the effects of similar physical forcings are usually compensated to $80-90 \%$ by dynamical feed-back processes, we see that, in this case, the compensation is kept below $10 \%$ so that the model nearly gets the full effect of the modification in its large scale features. In a certain sense one can therefore say that the model was "awaiting" this change to the parameterization of the PBL vertical fluxes.

Coming back to the discussion of Part III it is worth mentioning that these averaged effects bear a striking similarity to those diagnosed at ECMWF with a $K$-theory approach (this will not be shown here since we do not have any clear-cut comparison).

Since the effects on the moisture budget are far stronger than those on the energy budget we shall investigate the geographical

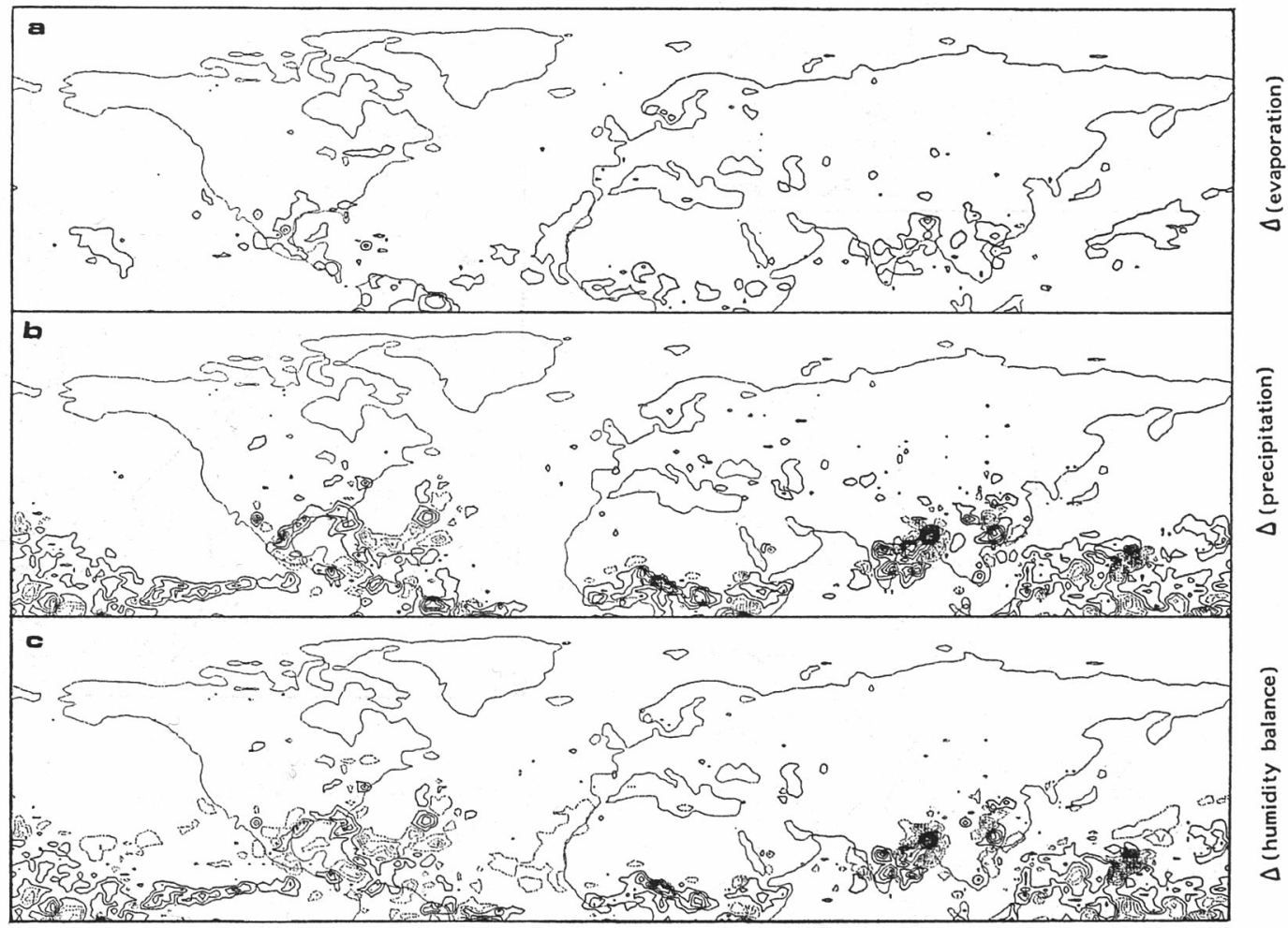

Fig. 5 Change to the surface evaporation (a), to the total rainfall (b) and to the net moisture budget (rainfall-evaporation) (c) due to shallow convection. Averaging period: day 3 to 4 of two forecasts starting from May the 16th 1986. Contour interval $2 \mathrm{~mm} /$ day. First contour at \pm 1 $\mathrm{mm} /$ day. Negative values are dotted. 
distribution of the shallow convection forcing through maps of its impact on surface evaporation (a), total precipitation (b) and net surface moisture balance $(c=b-a)$ (Figure 5). As expected there are only areas of enhanced evaporation. For precipitations the situation is more confused : there are areas of reinforcement associated with stronger evaporation over the continents (India, Africa and Amazonia); there are dipoles of positive and nega-
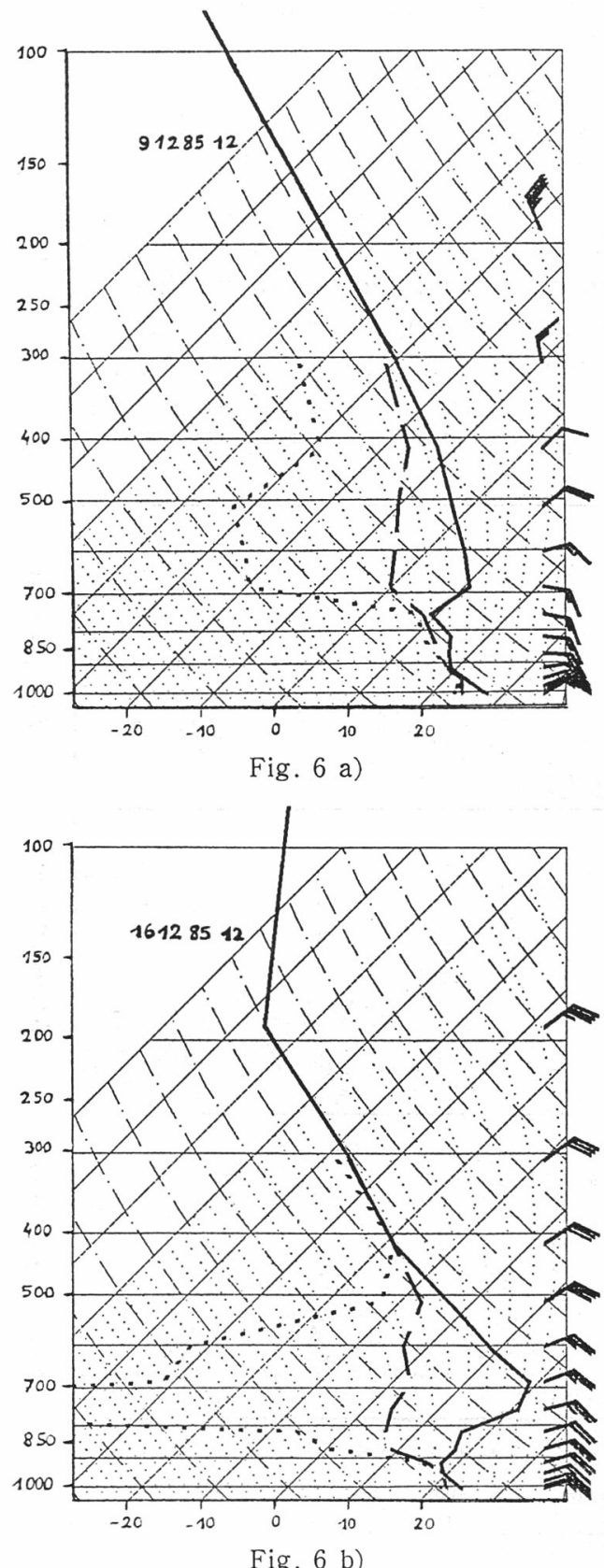

Fig. 6 b) tive increments indicating a displacement of rainfall areas through a modification of the tropical circulation (Western Pacific for example); and, more interestingly, there are signs of a systematic reinforcement of the ITCZ (Eastern Pacific and Eastern Atlantic). In Figure $5 c$ one can identify three areas where the supplementary evaporation is not immediately recycled by deep convection (a band stretching from California towards the West/ South West, near the African sub-tropical coast in the Atlantic and South East of Japan in the Western Pacific). At least for the first two of them, these are regions where one would expect to find shallow convection feeding moisture in the trade wind circulation, this moisture helping to maintain the ITCZ. It seems that the model is simulating just that through the parameterization that we are using.

Finally we also studied the impact of this parameterization in a one-dimensional model.

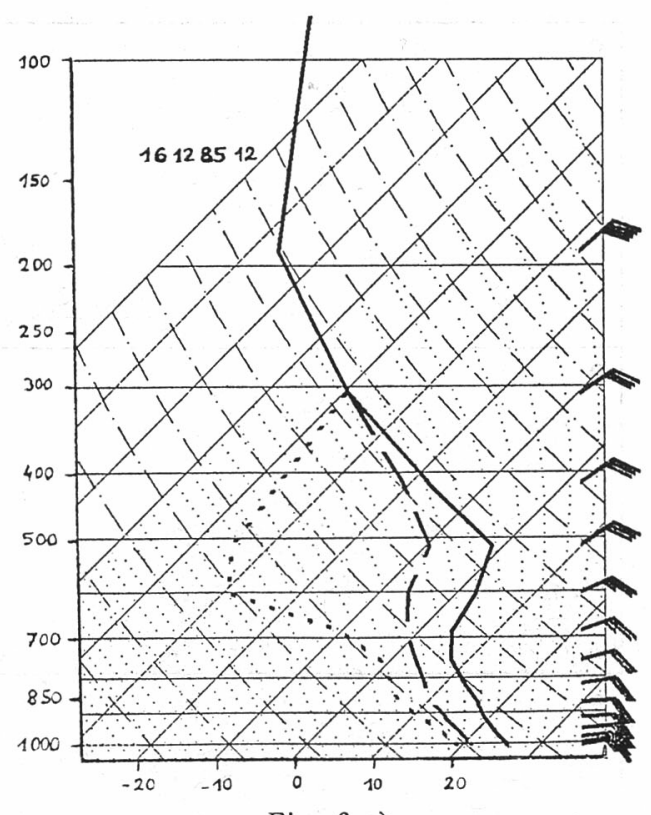

Fig. $6 \mathrm{c}$ )

Fig. 6 Tephigrams for a 1-D study of the effect of shallow convection.

a) original sounding

b) 7 days later without parameterization of shallow convection

c) 7 days later with parameterization of shallow convection.

Continuous line: $T$; Dashed line: $T_{w}$; Dotted line: $T_{d}$. 
Figure 6 shows three soundings, the first (a) (Barbados 9/12/85-12 h GMT) being used as initial conditions for two integrations with a $3 \mathrm{~mm} / \mathrm{s}$ externally prescribed subsidence, one without (b) and one with (c) parameterization of shallow convection. Results are shown after 7 days of integration. The PBL collapse is again obvious in Figure $6 \mathrm{~b}$ but in Figure $6 c$, despite a preservation of the general shape of the original sounding, one notices a rise of the PBL top from 750 to $700 \mathrm{hPa}$ connected to a smoothing of the inversion, and also some drying in the PBL. This may be attributed to the lack of vertical resolution of this 1-D modelization or to the unrealistic character of a sole downward vertical velocity as external forcing, but this also indicates a limitation of the scheme. Even if it works well in a full interactive configuration (cf. Fig. 3, 4 and 5) it cannot do more than what it was designed for: to parameterize in a simple way a process that the models cannot effectively simulate for lack both of a sufficient vertical resolution and of a prognostic liquid water variable.

The 1-D integration also reveals a tendency of the scheme (not shown here) to an on/off behaviour from one time step to the next with the leap-frog scheme. However this is not causing any difficulty in 3-D simulations with an Asselin-type time filter (avoided on purpose in the 1-D model) and with far more feed-back proccesses, except for the very first time-steps of a forecast before adaptation has taken place.

\section{Discussion}

The scheme presented here is a cheap, physically sound and efficient way of parameterizing the effects of shallow convection in large scale models concerned with tropical and subtropical areas. It obviously cannot replace a more detailed but far more expensive modelisation of the real physical proccesses occuring in PBL clouds but it offers a very adequate solution at to-day's level of sophistication of global and hemispheric NWP models.

\section{Acknowledgements}

The author is indebted to Ph. Bougeault for discussions before the design of the scheme, to A. Joly for its 1-D testing, to M. Tiedtke for his constructive comments on the manuscript and to P. Bonvalet for her expert typing.

\section{References}

Coiffier, J., Y. Ernie, JF. Geleyn, J. Clochard, J. Hoffman and F. Dupont, 1987: The operational hemispheric model at the French Meteorological Service. Short-and Medium-Range Numerical Weather Prediction, Collection of Paper Presented at the WMO/IUGG NWP Symposium, Tokyo, 4-8 August 1986, 337-345.

Imbard, M., R. Juvanon du Vachat, A. Joly, Y. Durand, JF. Geleyn, JM. Audoin, N. Marie and JM. Pairin, 1987: The Peridot fine-mesh numerical weather prediction system: description, evaluation and experiments. Short- and MediumRange Numerical Weather Prediction, Collection of Papers Presented at the WMO/IUGG NWP Symposium, Tokyo, 4-8 August 1986, 455-465.

Le Mone, M.A. and W. T. Pennell, 1976: The relationship of trade wind cumulus distribution to subcloud layer fluxes and structure. Mon. Wea. Rev., 104, 524-539.

Louis, Tiedtke and Geleyn, 1982: A short history of the PBL parameterization at ECMWF. Workshop on PBL parameterization. ECMWF Reading UK. 59-80.

Louis, JF., 1977: A case study of air mass transformation. ECMWF 1977 Seminar "The parameterization of the Physical Processes in the Free Atmosphere". ECMWF Reading UK. 357-373.

Tiedtke, 1982: Assessment of the PBL flow in the EC-model. Workshop on PBL parameterization. ECMWF Reading UK. 155-192.

- 1984: The sensitivity of the time-mean large-scale flow to cumulus convection in the ECMWF model. Workshop on convection in large-scale numerical models. ECMWF Reading UK. 297-316. 